\title{
PRIME IDEALS IN DIFFERENTIAL OPERATOR RINGS. CATENARITY
}

\author{
K. A. BROWN, K. R. GOODEARL AND T. H. LENAGAN
}

\begin{abstract}
Let $R$ be a commutative algebra over the commutative ring $k$, and let $\Delta=\left\{\delta_{1}, \ldots, \delta_{n}\right\}$ be a finite set of commuting $k$-linear derivations from $R$ to $R$. Let $T=R\left[\theta_{1}, \ldots, \theta_{n} ; \delta_{1}, \ldots, \delta_{n}\right]$ be the corresponding ring of differential operators. We define and study an isomorphism of left $R$-modules between $T$ and its associated graded ring $R\left[x_{1}, \ldots, x_{n}\right]$, a polynomial ring over $R$. This isomorphism is used to study the prime ideals of $T$, with emphasis on the question of catenarity. We prove that $T$ is catenary when $R$ is a commutative noetherian universally catenary $k$-algebra and one of the following cases occurs: (A) $k$ is a field of characteristic zero and $\Delta$ acts locally finitely; (B) $k$ is a field of positive characteristic; (C) $k$ is the ring of integers, $R$ is affine over $k$, and $\Delta$ acts locally finitely.
\end{abstract}

\section{INTRODUCTION}

Let the ring $R$ be an algebra over the commutative ring $k$, and let $\Delta=$ $\left\{\delta_{1}, \ldots, \delta_{n}\right\}$ be a finite set of commuting $k$-linear derivations from $R$ to itself. Let

$$
T=R\left[\theta_{1}, \ldots, \theta_{n} ; \delta_{1}, \ldots, \delta_{n}\right]
$$

be the corresponding ring of formal differential operators. (Thus the elements of $T$ may be written uniquely as left $R$-linear combinations of the ordered monomials in $\theta_{1}, \ldots, \theta_{n}$. Multiplication in $T$ is defined by extending the multiplication from $R$ according to the rules $\left[\theta_{i}, r\right]=\delta_{i}(r)$, for $r \in R$ and $i=1, \ldots, n$, and $\left[\theta_{i}, \theta_{j}\right]=0$, for $i, j=1, \ldots, n$.)

Our purpose here is to study the prime ideal structure of rings of this type, and, in particular, to investigate catenarity. (Recall that a ring $U$ is catenary if for any two prime ideals $P \subseteq Q$ in $U$, all saturated chains of prime ideals between $P$ and $Q$ have the same length. The ring $U$ is universally catenary provided that all finite polynomial extensions $U\left[x_{1}, \ldots, x_{n}\right]$ are catenary. All of the so-called excellent commutative noetherian rings are universally catenary [13, (34.A)]; in particular, commutative algebras affine over a field or over $\mathbb{Z}$ are universally catenary [13, Corollary $3,(14 . \mathrm{H})$ and Theorem $33,(16 . \mathrm{D})]$.$) An$ example constructed by Bell and Sigurdsson [2] shows that $T$ need not be catenary even when $n=1$ and $R$ is an affine $\mathbb{Q}$-algebra. However, in this example

Received by the editors February 18, 1988 and, in revised form, June 16, 1988.

1980 Mathematics Subject Classification (1985 Revision). Primary 16A05, 16 A66.

Key words and phrases. Differential operator ring, prime ideal, catenarity, height, derivation. 
$R$ contains an element $r$ such that the smallest $\Delta$-invariant $\mathbb{Q}$-subspace of $R$ containing $r$ has infinite dimension, and if such elements are forbidden, then positive results can be obtained. Indeed, Bell and Sigurdsson prove that if $k$ is a field of characteristic zero, $R$ is a commutative noetherian $k$-algebra, $\delta$ acts locally finitely on $R$, and the polynomial ring $R[x]$ is catenary, then $T$ is catenary [2].

We prove that $T$ is (universally) catenary in each of the following cases:

(A) $k$ is a field of characteristic zero, $R$ is a universally catenary commutative noetherian $k$-algebra, and $\Delta$ acts locally finitely on $R$ (Corollary 3.4);

(B) $k$ is a field of positive characteristic, and $R$ is a universally catenary commutative noetherian $k$-algebra (Corollary 4.7 );

(C) $k=\mathbb{Z}$, the ring $R$ is an affine commutative $k$-algebra, and $\Delta$ acts locally finitely on $R$ (Theorem 5.2).

In the affine case of case (B), and in case (C), we also prove that in each prime factor ring $T / P$, all maximal ideals have the same height (Theorems 4.8 and 5.2). Finally, we show that, at least in characteristic zero, the obstructions to catenarity in $T$ reside in the set of $\Delta$-prime ideals of $R$, in the following sense: if $R$ is a commutative noetherian $\mathbb{Q}$-algebra and $P \subseteq Q$ are prime ideals of $T$ with $P \cap R=Q \cap R$, then all saturated chains of prime ideals between $P$ and $Q$ have the same length (Corollary 6.2). In particular, if $R$ is a field of characteristic zero then $T$ is universally catenary (Theorem 6.1).

We proceed by defining an isomorphism $\Phi$ of left $R$-modules between $T$ and its associated graded ring $S=R\left[x_{1}, \ldots, x_{n}\right]$, a polynomial ring over $R$. This technique is particularly useful when $R$ is commutative. For in this case $S$ is commutative, and we show that there is a collection $\nabla$ of $k$-linear endomorphisms of $S$, containing $\Delta$, such that $\Phi$ defines a lattice isomorphism between the lattice of ideals of $T$ and the lattice of $\nabla$-invariants ideals of $S$ (Proposition 1.3).

To exploit this correspondence to study the prime ideals of $T$, one needs to study the ideals $\Phi(P)$ where $P$ is a prime ideal of $T$; these are the $\nabla$-prime ideals of $S$ (Theorem 1.7). The nature of the $\nabla$-prime ideals depends on the characteristic. Thus, if $T / P$ has characteristic zero, then $\Phi(P)$ is a prime ideal of $S$ (Theorem 2.3); but if $T / P$ has positive characteristic, then $\Phi(P)$ need not be prime (Examples 4.2 and 4.3).

The method of proof of the catenarity results thus also depends on the characteristic. In case $(\mathrm{A})$, we prove that there are "many" $\nabla$-invariant prime ideals of $S$ (Lemma 3.2), so that $S$ is catenary with respect to this set of prime ideals, whence the result follows. In case (B) we work instead with prime ideals of $S$ minimal over $\nabla$-prime ideals, showing that there are "many" of these prime ideals (Proposition 4.5); again, enough so that $S$ is catenary with respect to this set of prime ideals. In the special case of case (B) where $R$ is an affine $k$-algebra, $T$ is an affine PI-algebra (Theorem 4.1), and the catenarity of such rings was proved by Schelter [15]. It is not hard to splice these cases together to handle case $(\mathrm{C})$. 
The catenarity of $T$ in case (A) when $R$ is affine over $k$ admits an alternative proof. First, one reduces to the case where $K$ is algebraically closed (see [18]). In this case $T$ is a homomorphic image of an enveloping algebra $U(g)$ of a solvable Lie algebra $g$ of finite dimension over $k$. The catenarity of $U(g)$ was proved by Gabber [6;11, Corollary 9.8], and the catenarity of $T$ follows. We believe, however, that the proof presented here is of independent interest, being much more elementary than the proof just outlined and being an interesting application of the map $\Phi$.

The definition and basic general properties of $\Phi$ are given in $\S 1$. The proof that $\Phi(P)$ is prime when $T / P$ has characteristic zero is developed in $\S 2$. The applications to catenarity in cases (A), (B), and (C) are given in $\S \S 3,4$, and 5 respectively. In $\S 6$ we conclude with the partial catenarity results for pairs of prime ideals of $T$ with the same contraction to $R$.

This research was partially supported by a grant from the U.S. National Science Foundation, and was carried out while the authors visited the University of Washington in the summer of 1987. They thank these institutions for their support and hospitality.

\section{A CORRESPONDENCE BETWEEN DIFFERENTIAL OPERATOR IDEALS}

\section{AND POLYNOMIAL IDEALS}

Let $R$ be an algebra over a commutative ring $k$ and let $\Delta=\left\{\delta_{1}, \ldots, \delta_{n}\right\}$ be a finite set of commuting $k$-linear derivations on $R$. The elements of the formal differential operator ring

$$
T=R\left[\theta_{1}, \ldots, \theta_{n} ; \delta_{1}, \ldots, \delta_{n}\right]=R[\Theta ; \Delta]
$$

may be written uniquely as left $R$-linear combinations of the monomials in the $\theta$ 's. There is then an obvious left $R$-module isomorphism from $T$ to the ordinary polynomial ring

$$
S=R\left[x_{1}, \ldots, x_{n}\right]=R[X] .
$$

We intend to exploit this isomorphism in order to answer questions about the ideal structure of $T$. The first problem is to identify the subsets of $S$ that correspond to ideals of $T$.

In order to simplify the presentation of the calculations we will employ the following notation throughout this section.

\section{Notation N1(a).}

$R$ is an algebra over a commutative ring $k$.

$\delta_{1}, \ldots, \delta_{n}$ are commuting $k$-linear derivations on $R$.

$\Delta=\left\{\delta_{1}, \ldots, \delta_{n}\right\}$.

$T=R\left[\theta_{1}, \ldots, \theta_{n} ; \delta_{1}, \ldots, \delta_{n}\right]=R[\Theta ; \Delta]$.

$x_{1}, \ldots, x_{n}$ are independent commuting indeterminates.

$S=R\left[x_{1}, \ldots, x_{n}\right]=R[X]$. 
We extend each $\delta_{i}$ to derivations on $T$ and $S$ by setting $\delta_{i}\left(\theta_{j}\right)=0$ and $\delta_{i}\left(x_{j}\right)=0$ for all $i, j$. (Then $\delta_{i}(t)=\left[\theta_{i}, t\right]$ for all $t \in T$.) The following multi-index notations are used: for $I=(i(1), \ldots, i(n))$ in $\left(\mathbb{Z}^{+}\right)^{n}$ we set

$$
\theta^{I}=\theta_{1}^{i(1)} \theta_{2}^{i(2)} \cdots \theta_{n}^{i(n)} ; \quad x^{I}=x_{1}^{i(1)} x_{2}^{i(2)} \cdots x_{n}^{i(n)} ; \quad \delta^{I}=\delta_{1}^{i(1)} \delta_{2}^{i(2)} \cdots \delta_{n}^{i(n)} .
$$

In this notation, $T$ is a free left $R$-module with basis $\left\{\theta^{I}\right\}$ and $S$ is a free left $R$-module with basis $\left\{x^{I}\right\}$. Hence, there is a left $R$-module isomorphism $\Phi=\Phi_{R, \Delta}: T \rightarrow S$ such that $\Phi\left(\theta^{I}\right)=x^{I}$ for all $I$. (We omit the subscripts $R, \Delta$ as long as $T$ is the only differential operator ring under discussion.)

Lemma 1.1. (i) $\Phi(r t)=r \Phi(t)$ for all $r \in R$ and $t \in T$.

(ii) $\Phi\left(t \theta^{I}\right)=\Phi(t) x^{I}$ for all $t \in T$ and each multi-index $I$.

(iii) $\Phi\left(\left[\theta_{i}, t\right]\right)=\Phi \delta_{i}(t)=\delta_{i} \Phi(t)$ for all $i=1, \ldots, n$ and $t \in T$.

Proof. (i) and (ii) are clear from the definition of $\Phi$.

(iii) Since $\Phi$ is $k$-linear we need only check the result for $t=r \theta^{I}$ where $r \in R$ and $I$ is some multi-index. Then

$$
\Phi\left(\left[\theta_{i}, t\right]\right)=\Phi \delta_{i}(t)=\Phi\left(\delta_{i}(r) \theta^{I}\right)=\delta_{i}(r) x^{I}=\delta_{i}\left(r x^{I}\right)=\delta_{i} \Phi(t) .
$$

Part (iii) of Lemma 1.1 shows that bracketing by $\theta_{i}$ on $T$ corresponds to the action of $\delta_{i}$ on $S$. We next give a formal definition of the operator on $S$ that corresponds to the bracket $[r,-]$ on $T$ (for $r \in R$ ) and then list some properties. Except in computations with examples, an explicit description of this operator is not needed, and so we concentrate on its formal properties.

Notation N1(b). For $r \in R$, define a $k$-linear map $d_{r}: S \rightarrow S$ by the rule

$$
d_{r}(s)=\Phi\left(\left[r, \Phi^{-1}(s)\right]\right) .
$$

Therefore, $\Phi([r, t])=d_{r} \Phi(t)$ for all $r \in R$ and $t \in T$. We give the following lemma to illustrate some properties of $d_{r}$, although the formal definition of $d_{r}$ will suffice for our calculations.

Lemma 1.2. (i) $d_{r}\left(r^{\prime}\right)=\left[r, r^{\prime}\right]$ for all $r, r^{\prime} \in R$.

(ii) $d_{r}\left(x_{i}\right)=-\delta_{i}(r)$ for all $r \in R$ and $i=1, \ldots, n$.

(iii) $d_{r}\left(r^{\prime} s\right)=\left[r, r^{\prime}\right] s+r^{\prime} d_{r}(s)$ for all $r, r^{\prime} \in R$ and $s \in S$.

Proof. (i) $d_{r}\left(r^{\prime}\right)=d_{r} \Phi\left(r^{\prime}\right)=\Phi\left(\left[r, r^{\prime}\right]\right)=\left[r, r^{\prime}\right]$.

(ii) $d_{r}\left(x_{i}\right)=d_{r} \Phi\left(\theta_{i}\right)=\Phi\left(\left[r, \theta_{i}\right]\right)=\Phi\left(-\delta_{i}(r)\right)=-\delta_{i}(r)$.

(iii) Write $s=\Phi(t)$, for some $t \in T$. Then $r^{\prime} s=\Phi\left(r^{\prime} t\right)$ and

$$
\begin{aligned}
d_{r}\left(r^{\prime} s\right) & =d_{r} \Phi\left(r^{\prime} t\right)=\Phi\left(\left[r, r^{\prime} t\right]\right)=\Phi\left(\left[r, r^{\prime}\right] t+r^{\prime}[r, t]\right) \\
& =\left[r, r^{\prime}\right] \Phi(t)+r^{\prime} \Phi([r, t])=\left[r, r^{\prime}\right] s+r^{\prime} d_{r}(s) .
\end{aligned}
$$

Notation N1(c).

$$
\nabla=\Delta \cup\left\{d_{r} \mid r \in R\right\} .
$$

Thus $\nabla$ is a set of $k$-linear endomorphisms of $S$. 
Proposition 1.3. The map $\Phi: T \rightarrow S$ induces a lattice isomorphism between the lattice of ideals of $T$ and the lattice of $\nabla$-invariant left ideals of $S$.

Proof. Let $A$ be an ideal of $T$. Then $\Phi(A)$ is a left $R$-submodule of $S$. Also, if $a \in A$ then by Lemma 1.1(ii), $x_{i} \Phi(a)=\Phi(a) x_{i}=\Phi\left(a \theta_{i}\right) \in \Phi(A)$. Hence, $\Phi(A)$ is a left ideal of $S$. Next, if $a \in A$ then $\delta_{i} \Phi(a)=\Phi\left(\left[\theta_{i}, a\right]\right) \in \Phi(A)$ by Lemma 1.1(iii), and, if $r \in R$ then $d_{r} \Phi(a)=\Phi([r, a]) \in \Phi(A)$. Thus $\Phi(A)$ is a $\nabla$-invariant left ideal of $S$.

Conversely, let $B$ be a $\nabla$-invariant left ideal of $S$. It is immediate that $\Phi^{-1}(B)$ is closed under addition, left multiplication by elements of $R$, and right multiplication by each $\theta_{i}$. If $r \in R$ and $b \in B$, then

$$
\Phi^{-1}(b) r=r \Phi^{-1}(b)-\left[r, \Phi^{-1}(b)\right]=\Phi^{-1}(r b)-\Phi^{-1} d_{r}(b) \in \Phi^{-1}(B) .
$$

In addition, by Lemma 1.1,

$$
\begin{aligned}
\theta_{i} \Phi^{-1}(b) & =\left[\theta_{i}, \Phi^{-1}(b)\right]+\Phi^{-1}(b) \theta_{i}=\delta_{i} \Phi^{-1}(b)+\Phi^{-1}\left(b x_{i}\right) \\
& =\Phi^{-1}\left(\delta_{i}(b)+b x_{i}\right) \in \Phi^{-1}(B)
\end{aligned}
$$

for $i=1, \ldots, n$. Therefore $\Phi^{-1}(B)$ is an ideal of $T$.

When considering specific examples, the following lemma lessens the work involved in checking whether a left ideal of $S$ is invariant under $\nabla$.

Lemma 1.4. Let $B$ be a left ideal of $S$, and let $G$ generate $R$ as a $k$-algebra. If $d_{g}(B) \subseteq B$ for all $g \in G$, then $d_{r}(B) \subseteq B$ for all $r \in R$.

Proof. Observe that $\Phi^{-1}(B)$ is a left $R$-submodule of $T$. Since $B$ is closed under $d_{g}$ for all $g \in G$, it follows that $\Phi^{-1}(B)$ is closed under bracketing with elements of $G$, whence $\Phi^{-1}(B)$ is closed under right multiplication by elements of $G$. Since $G$ generates $R$ as a $k$-algebra, $\Phi^{-1}(B)$ must be a right $R$-submodule of $T$. Thus $\Phi^{-1}(B)$ is closed under bracketing with elements of $R$, and therefore $B$ is closed under $d_{r}$ for all $r \in R$.

For the rest of the section, we concentrate on the case where $R$ is a commutative ring. In this case $S$ is a commutative ring and so Proposition 1.3 gives a lattice isomorphism between the ideals of $T$ and the $\nabla$-invariant ideals of $S$. We abbreviate the latter to $\nabla$-ideals.

Given any set $D$ of maps from a ring $A$ to itself, the $D$-invariant ideals of $A$ are called $D$-ideals. A $D$-prime ideal of $A$ is any proper $D$-ideal $P$ such that whenever $I, J$ are $D$-ideals of $A$ satisfying $I J \subseteq P$ then either $I \subseteq P$ or $J \subseteq P$. (It is enough to check this for $D$-ideals $I, J$ that contain $P$.) Finally, $A$ is called a $D$-prime ring if 0 is a $D$-prime ideal of $A$.

We use this terminology in several different cases, with either $D=\nabla$ or $D=\Delta$.

The main aim of the rest of the section is to show that when $R$ is commutative the map $\Phi$ induces a bijection between the prime ideals of $T$ and the 
$\nabla$-prime ideals of $S$. In the following section we are able to show, after developing some further machinery, that in case $R$ is a commutative noetherian Q-algebra the $\nabla$-prime ideals of $S$ are in fact prime ideals.

If $\theta^{I}$ is any monomial in $T$ and $I=(i(1), \ldots, i(n))$ then the total degree of $\theta^{I}$ is the nonnegative integer $|I|=i(1)+\cdots+i(n)$. The total degree of an element $t=\sum a_{I} \theta^{I}$ in $T$, denoted $\operatorname{deg}(t)$, is the maximal total degree of the monomials with nonzero coefficients $a_{I}$. The same definition applies for the total degree of elements of $S$. In both cases, we observe the convention that $\operatorname{deg}(0)=-\infty$. Note that $\Phi$ preserves total degree.

Lemma 1.5. Let $R$ be commutative and $r \in R$.

(i) $\operatorname{deg}([r, t])<\operatorname{deg}(t)$, for all nonzero $t \in T$.

(ii) $\operatorname{deg}\left(d_{r}(s)\right)<\operatorname{deg}(s)$, for all nonzero $s \in S$.

Proof. (i) It is enough to check that $\operatorname{deg}\left(\left[r, a \theta^{I}\right]\right)<|I|$, for any nonzero $a \in R$ and any multi-index $I$. If $I=0$ the result holds since $R$ is commutative. If $I \neq 0$ choose an index $j$ with $i(j) \neq 0$, and let $J$ be the multi-index that is the same as $I$ except in the $j$ th position, where the entry is reduced by one. Then

$$
\left[r, a \theta^{I}\right]=a\left[r, \theta_{j} \theta^{J}\right]=a \theta_{j}\left[r, \theta^{J}\right]+a\left[r, \theta_{j}\right] \theta^{J} .
$$

The result now follows by induction on total degree since $|J|<|I|$ and $\left[r, \theta_{j}\right]=$ $-\delta_{j}(r) \in R$.

(ii) follows from (i) and the definition of $d_{r}$, since the map $\Phi$ preserves total degree.

Proposition 1.6. Let $R$ be commutative, let $A$ be an ideal of $T$, and set $B=$ $\Phi(A)$. Set

$$
C_{1}=\{c \in T \mid[c, R] \subseteq A\}, \quad C_{2}=\left\{c \in S \mid d_{r}(c) \in B, \text { for all } r \in R\right\} .
$$

(i) $C_{1} / A$ is a subalgebra of $T / A$ and $C_{2} / B$ is a subalgebra of $S / B$.

(ii) $\Phi\left(C_{1}\right)=C_{2}$.

(iii) $\boldsymbol{\Phi}(c t)-\boldsymbol{\Phi}(c) \Phi(t) \in B$, for all $c \in C_{1}$ and $t \in T$.

(iv) $\Phi$ induces a $k$-algebra isomorphism from $C_{1} / A$ onto $C_{2} / B$.

Proof. (i) Clearly, $C_{1} / A$ is the centralizer in $T / A$ of $(R+A) / A$, and so is a subalgebra of $T / A$. That $C_{2} / B$ is a subalgebra of $S / B$ will be clear once (ii) and (iii) are proved.

(ii) If $c \in T$ and $r \in R$ then $d_{r} \Phi(c)=\Phi([r, c])$; hence, $d_{r} \Phi(c) \in B$ if and only if $[r, c] \in A$. That is, $\Phi(c) \in C_{2}$ if and only if $c \in C_{1}$.

(iii) Let $c \in C_{1}$ and $t \in T$, and write $c=\sum c_{I} \theta^{I}$ and $t=\sum t_{J} \theta^{J}$, where $c_{I}, t_{J} \in R$ and all but finitely many $c_{I}, t_{J}$ are zero. Working modulo $A$ we see that $c t_{J} \equiv t_{J} c$ for all $J$ (because $c \in C_{1}$ ) and so

$$
c t=\sum_{J} c t_{J} \theta^{J} \equiv \sum_{J} t_{J} c \theta^{J}=\sum_{I, J} t_{J} c_{I} \theta^{I+J} .
$$


Hence, working modulo $B=\Phi(A)$,

$$
\Phi(c t) \equiv \Phi\left(\sum_{I, J} t_{J} c_{I} \theta^{I+J}\right)=\sum_{I, J} t_{J} c_{I} x^{I+J}=\Phi(c) \Phi(t) .
$$

(iv) is clear from (ii) and (iii).

Theorem 1.7. Let $R$ be a commutative k-algebra, let $A$ be an ideal of $T$, and set $B=\Phi(A)$. Set

$$
C_{1}=\{c \in T \mid[c, R] \subseteq A\}, \quad C_{2}=\left\{c \in S \mid d_{r}(c) \in B, \text { for all } r \in R\right\} .
$$

Then the following conditions are equivalent:

(i) $A$ is a prime ideal of $T$.

(ii) $C_{1} / A$ is a $\Delta$-prime ring.

(iii) $C_{2} / B$ is a $\Delta$-prime ring.

(iv) $B$ is a $\nabla$-prime ideal of $S$.

Proof. Let $c \in C_{1}$ and $r \in R$. Then

$$
\left[\delta_{i}(c), r\right]=\left[\left[\theta_{i}, c\right], r\right]=-\left[[c, r], \theta_{i}\right]-\left[\left[r, \theta_{i}\right], c\right] \in\left[A, \theta_{i}\right]+[R, c] \subseteq A .
$$

Thus $C_{1}$ is invariant under $\Delta$. Since $\Phi\left(C_{1}\right)=C_{2}$ by Proposition 1.6(ii), and since $\Phi$ preserves the action of $\Delta$ by Lemma 1.1(iii), it follows that $C_{2}$ is invariant under $\Delta$. As $A$ and $B$ are invariant under $\Delta$, the rings $C_{1} / A$ and $C_{2} / B$ inherit actions of $\Delta$.

(ii) $\Leftrightarrow$ (iii): By Proposition 1.6(iv), $\Phi$ induces a ring isomorphism of $C_{1} / A$ onto $C_{2} / B$, and $\Phi$ preserves the action of $\Delta$. Thus $C_{1} / A$ is a $\Delta$-prime ring if and only if $C_{2} / B$ is a $\Delta$-prime ring.

(i) $\Rightarrow$ (ii): Let $E, F$ be $\Delta$-ideals of $C_{1}$ that properly contain $A$. Now $[E, R] \subseteq A \subseteq E$ and so $R E=E R$. Also, $\left[\theta_{i}, E\right] \subseteq E$ for $i=1, \ldots, n$, so that $\theta_{i} E+E=E \theta_{i}+E$, and therefore $T E=E T$. Similarly, $T F=F T$. Hence, $T E$ and $F T$ are ideals of $T$ that properly contain $A$. Since $A$ is a prime ideal, $T E F T \nsubseteq A$, and so $E F \nsubseteq A$.

(ii) $\Rightarrow$ (i): Let $E, F$ be ideals of $T$ properly containing $A$. Set $H=E \cap C_{1}$ and $K=F \cap C_{1}$, and note that $H$ and $K$ are $\Delta$-ideals of $C_{1}$. Choose an element $t \in E-A$ with $\operatorname{deg}(t)$ as small as possible. By Lemma 1.5(i), $\operatorname{deg}([r, t])<\operatorname{deg}(t)$ for all $r \in R$; hence, $[t, R] \subseteq A$. Thus $t \in H$ and $H$ properly contains $A$. Similarly, $K$ properly contains $A$; hence, $H K \nsubseteq A$, since $A$ is a $\Delta$-prime ideal of $C_{1}$. Therefore $E F \nsubseteq A$.

(iii) $\Rightarrow$ (iv) is similar to (ii) $\Rightarrow$ (i), using Lemma 1.5(ii) in place of Lemma 1.5(i).

(iv) $\Rightarrow$ (iii): Let $E$ be a $\Delta$-ideal of $C_{2}$ containing $B$. Set $H=\Phi^{-1}(E)$, so that $H$ is a $\Delta$-ideal of $C_{1}$ containing $A$. As in the proof of (i) $\Rightarrow$ (ii), it follows that $H T=T H$ is an ideal of $T$. Thus, by Proposition 1.3, $\Phi(H T)$ is a $\nabla$-ideal of $S$. Now, since $H \subseteq C_{1}$ and $B \subseteq E$, Proposition 1.6(iii) implies that $\Phi(H T)=E S$. Hence, $E S$ is a $\nabla$-ideal of $S$. 
Suppose that $E, F$ are $\Delta$-ideals of $C_{2}$ properly containing $B$. Then, by the preceding paragraph, $E S$ and $F S$ are $\nabla$-ideals of $S$, and they properly contain $B$. Thus, since $B$ is a $\nabla$-prime ideal of $S$, we obtain $E S F S \nsubseteq B$ and so $E F \nsubseteq B$. Therefore $C_{2} / B$ is a $\Delta$-prime ring.

In the next section we will show that when $R$ is a commutative noetherian Q-algebra the $\nabla$-prime ideals of $S$ are prime; hence, for any prime ideal $P$ of $T$ the image $\Phi(P)$ is a prime ideal of $S$.

Corollary 1.8. Let $R$ be a commutative ring equipped with commuting derivations $\delta_{1}, \ldots, \delta_{n}$, set $T=R\left[\theta_{1}, \ldots, \theta_{n} ; \delta_{1}, \ldots, \delta_{n}\right]$ and $S=R\left[x_{1}, \ldots, x_{n}\right]$, and define $\Delta, \nabla$ as in Notation N1. Then the map $\Phi_{R, \Delta}$ induces a bijection between the set of prime ideals of $T$ and the set of $\nabla$-prime ideals of $S$; moreover $\Phi_{R, \Delta}$ and its inverse both preserve inclusions.

Proof. Proposition 1.3 and Theorem 1.7.

If $R$ is commutative and $A$ is a prime ideal of $T$, then by Theroem 1.7 $S / \Phi(A)$ is a $\nabla$-prime ring-the product of any two nonzero $\nabla$-ideals of $S / \Phi(A)$ is nonzero. Since it is not clear whether the annihilator of a $\nabla$-ideal is itself a $\nabla$-ideal, it does not immediately follow that nonzero $\nabla$-ideals in $S / \Phi(A)$ have zero annihilator. However, this is true, as we now show.

Proposition 1.9. Let $R$ be commutative and let $B$ be a $\nabla$-prime ideal of $S$. Then any nonzero $\nabla$-ideal of $S / B$ has zero annihilator in $S / B$.

Proof. Set $A=\Phi^{-1}(B)$, which by Theorem 1.7 is a prime ideal of $T$. Let $F$ be a $\nabla$-ideal of $S$ properly containing $B$; we must show that $\operatorname{ann}_{S / B}(F / B)=0$.

Now $E=\Phi^{-1}(F)$ is an ideal of $T$ properly containing $A$. Let $d$ be the minimal degree for elements of $E-A$, and let $C$ be the set of those elements of $E$ having degree $d$. If $c \in C$ and $r \in R$, then $[c, r] \in E$ and $\operatorname{deg}([c, r])<d$ by Lemma $1.5(\mathrm{i})$, whence $[c, r] \in A$. Thus $[C, R] \subseteq A$. Moreover, $\left[\theta_{i},-\right]$, for $i=1, \ldots, n$, cannot raise degree, from which we see that $\left[\theta_{i}, C\right] \subseteq C \cup A$. It follows that $T C+A$ is an ideal of $T$. Since $A$ is a prime ideal, the right annihilator of $C$ in $T / A$ is thus zero.

If $s \in S$ and $F s \subseteq B$, then $\Phi(C) s \subseteq B$. Since $[C, R] \subseteq A$, Proposition 1.6(iii) shows that $C \Phi^{-1}(s) \subseteq A$, whence $\Phi^{-1}(s) \in A$ and $s \in B$. Therefore $\operatorname{ann}_{S / B}(F / B)=0$, as desired.

Corollary 1.10. Let $R$ be commutative noetherian. If $Q$ is a prime ideal of $T$ and $H$ is a prime ideal of $S$ that is minimal over $\Phi(Q)$, then $\Phi(Q)$ is the largest $\nabla$-ideal of $S$ that is contained in $H$.

Proof. By Theorem 1.7, $\Phi(Q)$ is a $\nabla$-prime ideal of $S$. Since $H / \Phi(Q)$ is a minimal prime ideal in the commutative noetherian ring $S / \Phi(Q)$, its annihilator must be nonzero. Thus, by Proposition 1.9, $H / \Phi(Q)$ contains no nonzero $\nabla$-ideals of $S / \Phi(Q)$. 


\section{D-PRIME IDEALS IN CHARACTERISTIC ZERO}

Our aim in this section is to complete the characterization of the $\nabla$-prime ideals appearing in the $\Phi$-correspondence (Theorem 1.7 and Corollary 1.8), in the case that the coefficient ring $R$ is a commutative noetherian $\mathbb{Q}$-algebra. In this case we show that the $\nabla$-prime ideals in the polynomial ring $S$ are precisely the prime $\nabla$-ideals of $S$.

Throughout the section we continue to fix Notation N1 as in the previous section. The first tool we develop is an analog of Proposition 1.6.

Proposition 2.1. Let $R$ be commutative, let $A$ be an ideal of $T$, and set $B=$ $\Phi(A)$. Set

$$
\begin{aligned}
& C_{1}=\left\{c \in T \mid \delta_{i}(c) \in A, \text { for } i=1, \ldots, n\right\}, \\
& C_{2}=\left\{c \in S \mid \delta_{i}(c) \in B, \text { for } i=1, \ldots, n\right\} .
\end{aligned}
$$

(i) $C_{1} / A$ is a subalgebra of $T / A$ and $C_{2} / B$ is a subalgebra of $S / B$.

(ii) $\Phi\left(C_{1}\right)=C_{2}$.

(iii) $\Phi(t c)-\Phi(t) \Phi(c) \in B$, for all $t \in T$ and $c \in C_{1}$.

(iv) $\Phi$ induces a k-algebra isomorphism from $C_{1} / A$ onto $C_{2} / B$.

Proof. (i) and (ii) are clear, and (iv) will follow from (ii) and (iii).

(iii) Let $t \in T$ and $c \in C_{1}$ and write $t=\sum t_{I} \theta^{I}$ and $c=\sum c_{J} \theta^{J}$, where $t_{I}, c_{J} \in R$ and all but finitely many $t_{I}, c_{J}$ are zero. Working modulo $A$, we see that $\theta_{i} c \equiv c \theta_{i}$ for $i=1, \ldots, n$ (because $c \in C_{1}$ ) and so $\theta^{I} c \equiv c \theta^{I}$ for all $I$, whence

$$
t c=\sum_{I} t_{I} \theta^{I} c \equiv \sum_{I} t_{I} c \theta^{I}=\sum_{I, J} t_{I} c_{J} \theta^{I+J} .
$$

Hence, working modulo $B=\Phi(A)$,

$$
\Phi(t c) \equiv \sum_{I, J} t_{I} c_{J} x^{I+J}=\Phi(t) \Phi(c) .
$$

In order to utilize Proposition 2.1 effectively, we need to be able to find suitable elements $c \in S$ such that $\delta_{i}(c) \in B$ for all $i$. Unlike our use of Proposition 1.6 (in the proof of Theorem 1.7), it is not enough to look for elements in $S-B$ of minimal total degree, since the $\delta_{i}$ do not usually lower total degree. Instead, we introduce a finer notion of degree, with respect to which leading coefficients can be defined, and we shall be able to use Proposition 2.1 by finding elements in $S-B$ whose new degree is minimal and which have leading coefficient 1 ; then applying the $\delta_{i}$ does lower the degree.

We now fix the following Dixmier ordering on our multi-indices in $\left(\mathbb{Z}^{+}\right)^{n}$. Given any multi-indices $I$ and $J$, we set $I<J$ in the Dixmier ordering if and only if either $|I|<|J|$ or else $|I|=|J|$ and $I$ is lexicographically less than $J$ (that is, $I \neq J$ and if $m$ is the smallest index for which $i(m) \neq j(m)$, then $i(m)<j(m))$. Observe that $\left(\mathbb{Z}^{+}\right)^{n}$ equipped with the Dixmier ordering is well-ordered (in fact, it is order-isomorphic to $\mathbb{Z}^{+}$). Hence, with respect to this 
ordering on the multi-indices, we may define degrees and leading coefficients for polynomials in $S$ and operators in $T$ in the usual fashion.

Lemma 2.2. Let $R_{0}$ be a noetherian ring, $\Delta_{0}$ a set of derivations acting on $R_{0}$, and $P_{0}$ a $\Delta_{0}$-prime ideal of $R_{0}$.

(i) There is exactly one prime ideal in $R_{0}$ minimal over $P_{0}$.

(ii) If $\operatorname{char}\left(R_{0} / P_{0}\right)=0$, then $P_{0}$ is a prime ideal.

Proof. Apply [5, Lemma 2 and Corollary] to the $\Delta_{0}$-prime ring $R_{0} / P_{0}$. (The lemma in fact holds if $R_{0}$ is only assumed to be right noetherian. For a proof in the case where $\Delta_{0}$ consists of a single derivation, see [9, Lemma 2.1 and Theorem 2.2] or [7, Proposition 1.5].)

Theorem 2.3. Let $R$ be commutative noetherian. If $P$ is a prime ideal of $T$ such that $\operatorname{char}(T / P)=0$, then $\Phi(P)$ is a prime ideal of $S$.

Proof. Note that $P \cap R$ is a $\Delta$-prime ideal of $R$. There is a natural commutative diagram as follows:

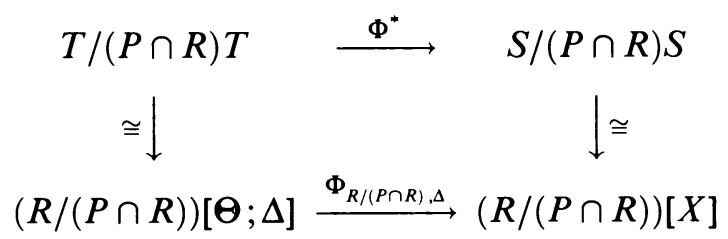

where $\Phi^{*}$ is the map induced by $\Phi_{R, \Delta}$. It is enough to show that the ideal $\Phi^{*}(P /(P \cap R) T)$ is a prime ideal of $S /(P \cap R) S$, and hence it is enough to show that the map $\Phi_{R /(P \cap R), \Delta}$ carries the image of $P /(P \cap R) T$ to a prime ideal of $(R /(P \cap R))[X]$. Thus, without loss of generality, $P \cap R=0$.

Now $R$ is a $\Delta$-prime ring of characteristic zero and so $R$ is a domain (Lemma 2.2). Let $K$ be the quotient field of $R$, and extend the action of $\Delta$ to $K$ via the quotient rule. The set $R-\{0\}$ is an Ore set in $T$ and in $S$, and we may denote the corresponding localizations by $K T$ and $K S$. By [3, Satz 2.10], $K P$ is a prime ideal of $K T$ and $K P \cap T=P$; in particular, it follows that $T / P$ is torsionfree as a left $R$-module, and hence $S / \Phi_{R, \Delta}(P)$ is torsionfree as a left $R$-module. Consequently, $K \Phi_{R, \Delta}(P) \cap S=\Phi_{R, \Delta}(P)$, and so it suffices to show that $K \Phi_{R, \Delta}(P)$ is a prime ideal of $K S$.

If we identify $K T$ with $K[\Theta ; \Delta]$ and $K S$ with $K[X]$, we find that $K \Phi_{R, \Delta}(P)$ $=\Phi_{K, \Delta}(K P)$, and so it is enough to show that the latter is a prime ideal of $K[X]$. Thus we may, without loss of generality, assume that $R$ is a field.

Since $R$ is now in particular a commutative noetherian $\mathbb{Q}$-algebra, [16, Corollary 2.6] shows that every prime ideal of $T$ is completely prime. Thus $T / P$ is a domain.

Set $B=\Phi(P)$. In view of Lemma 2.2 , it is enough to show that $B$ is a $\Delta$-prime ideal of $S$. Hence, let $E, F$ be any two $\Delta$-ideals of $S$ not contained in $B$; we need to show that $E F \nsubseteq B$. 
Let $I$ be the minimal degree (with respect to the Dixmier ordering) for elements of $F-B$, and choose $f \in F-B$ with degree $I$. Since $R$ is a field, we may multiply $f$ by the inverse of its leading coefficient; hence, there is no loss of generality in assuming that the leading coefficient of $f$ is 1 . Now for $i=1, \ldots, n$ we observe that $\delta_{i}(f)$ has degree less than $I$. As $F$ is a $\Delta$-ideal, $\delta_{i}(f) \in F$, and so $\delta_{i}(f) \in B$ by the minimality of $I$.

If $c=\Phi^{-1}(f)$, then $c \in T-P$ and $\delta_{i}(c) \in P$ for $i=1, \ldots, n$. Choose an element $t$ in $\Phi^{-1}(E)-P$. Since $T / P$ is a domain, $t c \notin P$. Consequently, by Proposition 2.1(iii), $\Phi(t) f \notin B$, and therefore $E F \nsubseteq B$, as desired.

Corollary 2.4. Assume that $R$ is a commutative noetherian Q-algebra.

(a) The $\nabla$-prime ideals of $S$ are precisely the prime $\nabla$-ideals.

(b) If $I$ is a $\nabla$-ideal of $S$ and $P$ is a prime ideal minimal over $I$, then $P$ is a $\nabla$-ideal.

Proof. (a) Obviously any prime $\nabla$-ideal of $S$ is a $\nabla$-prime ideal. Conversely, if $B$ is a $\nabla$-prime ideal of $S$ then $\Phi^{-1}(B)$ is a prime ideal of $T$ by Theorem 1.7, and therefore $B$ is a prime ideal of $S$ by Theorem 2.3.

(b) Let $Q$ be the largest $\nabla$-ideal contained in $P$; then $I \subseteq Q \subseteq P$. If $E, F$ are $\nabla$-ideals such that $E F \subseteq Q$ then $E F \subseteq P$, and so either $E \subseteq P$ or $F \subseteq P$. Thus either $E$ or $F$ is a $\nabla$-ideal contained in $P$, whence either $E \subseteq Q$ or $F \subseteq Q$. This shows that $Q$ is a $\nabla$-prime ideal, and hence $Q$ is a prime ideal, by (a). But $P$ is minimal over $I$, and so $Q=P$. Therefore $P$ is a $\nabla$-ideal.

Combining Corollary 2.4 with Corollary 1.8 yields the best case of the $\Phi$ correspondence, as follows.

Theorem 2.5. Let $R$ be a commutative noetherian $\mathbb{Q}$-algebra equipped with commuting derivations $\delta_{1}, \ldots, \delta_{n}$, set $T=R\left[\theta_{1}, \ldots, \theta_{n} ; \delta_{1}, \ldots, \delta_{n}\right]$ and $S=$ $R\left[x_{1}, \ldots, x_{n}\right]$, and define $\Delta, \nabla$ as in Notation N1. Then the map $\Phi_{R, \Delta}$ induces a bijection between the set of prime ideals of $T$ and the set of prime $\nabla$-ideals of $S$; moreover $\Phi_{R, \Delta}$ and its inverse both preserve inclusions.

\section{Catenarity IN CHARACTERISTIC ZeRO}

The aim in this section is to investigate catenarity for a differential operator ring $T$ as in Notation N1. Assuming $k$ is a field of characteristic zero, $R$ is a universally catenary commutative noetherian $k$-algebra, and $\Delta$ acts locally finitely on $R$, we shall prove that $T$ is universally catenary. The necessity of the locally finite hypothesis is shown by an example of Bell and Sigurdsson [2, Example 2.10]. We consider catenarity results in positive and mixed characteristics in later sections.

Our approach in the present case is to use the $\Phi$-correspondence of $\S \S 1$ and 2 to transfer the problem to the polynomial ring $S$. Given prime ideals $P \subseteq Q$ in $T$, we know that $\Phi(P) \subseteq \Phi(Q)$ are prime $\nabla$-ideals of $S$ (Theorem 2.3), 
and we shall show that any saturated chain of prime $\nabla$-ideals between $\Phi(P)$ and $\Phi(Q)$ is in fact a saturated chain of prime ideals. Once this is done the catenarity of $T$ follows from that of $S$. This approach owes something to arguments of Lorenz [12].

In this section we fix the following notation.

\section{Notation N2.}

$k$ is a field.

$R$ is a commutative $k$-algebra.

In addition, Notation $\mathrm{N} 1$ will be in effect throughout the section, and we will compute degrees in $T$ and $S$ relative to the Dixmier ordering introduced in $\S 2$.

The set $\Delta$ of derivations is said to act locally finitely on $R$ provided each element of $R$ is contained in a finite-dimensional $k$-subspace which is invariant under $\Delta$.

Lemma 3.1. Suppose that $\Delta$ acts locally finitely on $R$, and let $A, B$ be $\nabla$-ideals of $S$ with $A \nsubseteq B$.

(i) If $k$ is algebraically closed, there exists $a \in A-B$ such that $k a+B$ is invariant under $\Delta$ and $d_{r}(a) \in B$ for all $r \in R$.

(ii) For any $a \in A$ as in (i), the set $B+S a$ is a $\nabla$-ideal of $S$.

Proof. (i) After replacing $B$ by $B \cap A$, we may assume that $B$ is properly contained in $A$. Let $I$ be the minimal degree (with respect to the Dixmier ordering) for elements of $A-B$, and choose $c \in A-B$ with degree $I$ and leading coefficient $\gamma$. Define

$$
\begin{aligned}
& A^{\circ}=\{0\} \cup\{\text { leading coefficients of elements of } A \text { of degree } I\}, \\
& B^{\circ}=\{0\} \cup\{\text { leading coefficients of elements of } B \text { of degree } I\} .
\end{aligned}
$$

It is clear that $B^{\circ} \subseteq A^{\circ}$ and that these are $\Delta$-ideals of $R$. Then $\gamma \in A^{\circ}$ by definition of $A^{\circ}$, and $\gamma \notin B^{\circ}$ since if there existed $b \in B$ with degree $I$ and leading coefficient $\gamma$, then $c-b$ would be an element of $A-B$ with degree less than $I$.

Since $\Delta$ acts locally finitely on $R$, there exists a finite-dimensional $\Delta$-invariant $k$-subspace $V \subseteq R$ such that $\gamma \in V$. After replacing $V$ by $V \cap A^{\circ}$, we may assume that $V \subseteq A^{\circ}$. Since $k$ is algebraically closed and $\Delta$ acts as a finite set of commuting $k$-linear transformations on $\left(V+B^{\circ}\right) / B^{\circ}$, there must exist $\alpha \in V-B^{\circ}$ such that the coset $\alpha+B^{\circ}$ is a $\Delta$-eigenvector. Hence, $\alpha \in A^{\circ}-B^{\circ}$ and there exist $\lambda_{1}, \ldots, \lambda_{n} \in k$ such that $\delta_{i}(\alpha)-\lambda_{i} \alpha \in B^{\circ}$ for $i=1, \ldots, n$.

Choose $a \in A$ with degree $I$ and leading coefficient $\alpha$. Then $a \notin B$ because $\alpha \notin B^{\circ}$. For $i=1, \ldots, n$ observe that

$$
\delta_{i}(a)-\lambda_{i} a=\left(\delta_{i}(\alpha)-\lambda_{i} \alpha\right) x^{I}+\text { [terms of lower degree]. }
$$

Since $\delta_{i}(\alpha)-\lambda_{i} \alpha \in B^{\circ}$, there exists $b_{i} \in B$ such that

$$
b_{i}=\left(\delta_{i}(\alpha)-\lambda_{i} \alpha\right) x^{I}+[\text { terms of lower degree] } .
$$


Then $\delta_{i}(a)-\lambda_{i} a-b_{i}$ is an element of $A$ with degree less than $I$, whence $\delta_{i}(a)-\lambda_{i} a-b_{i} \in B$, and hence $\delta_{i}(a)-\lambda_{i} a \in B$. As $B$ is $\Delta$-invariant, it follows that the set $k a+B$ is invariant under $\Delta$.

For $r \in R$, Lemma 1.5(ii) shows that $d_{r}(a)$ has lower total degree than $a$, whence $d_{r}(a)$ has Dixmier degree less than $I$. Since $A$ is $\nabla$-invariant, $d_{r}(a) \in A$, and then $d_{r}(a) \in B$ by minimality of $I$.

(ii) Set $c=\Phi^{-1}(a)$. Since $k a+B$ is invariant under $\Delta$, so is $k c+\Phi^{-1}(B)$, and since $d_{r}(a) \in B$ for all $r \in R$, we obtain $[c, R] \subseteq \Phi^{-1}(B)$. Thus $k c+\Phi^{-1}(B)$ is also invariant under $[-, R]$.

It follows that $c T+\Phi^{-1}(B)$ is an ideal of $T$. As $[c, R] \subseteq \Phi^{-1}(B)$, we conclude from Proposition 1.6(iii) that $\Phi\left(c T+\Phi^{-1}(B)\right)=a S+B$. Therefore $a S+B$ is a $\nabla$-ideal of $S$, by Proposition 1.3.

Given prime $\nabla$-ideals $P \subseteq Q$ in $S$, the $\nabla$-height of $Q / P$, denoted $\nabla-\mathrm{ht}(Q / P)$, is defined to be the supremum of the lengths of all chains of prime $\nabla$-ideals between $P$ and $Q$.

Lemma 3.2. Let $\operatorname{char}(k)=0$, let $R$ be noetherian, and let $\Delta$ act locally finitely on $R$. If $P \subseteq Q$ are prime $\nabla$-ideals of $S$ such that $\nabla \cdot \mathrm{ht}(Q / P)=1$, then $\operatorname{ht}(Q / P)=1$.

Proof. Let $k^{\#}$ denote the algebraic closure of $k$, and set $R^{\#}=R \otimes_{k} k^{\#}$ and $S^{\#}=S \otimes_{k} k^{\#}$. View $R$ and $S$ as subalgebras of $R^{\#}$ and $S^{\#}$; then in particular $S^{\#}$ is an integral extension of $S$. Now identify $S^{\#}$ with $R^{\#}\left[x_{1}, \ldots, x_{n}\right]$ and let $\nabla^{\#}$ denote the set $\Delta \cup\left\{d_{r} \mid r \in R^{\#}\right\}$ of $k^{\#}$-linear operators on $S^{\#}$. For $r \in R$ the operator $d_{r}$ on $S^{\#}$ is the natural $k^{\#}$-linear extension of the operator $d_{r}$ on $S$, and so we may identify $\nabla$ with a subset of $\nabla^{\#}$; then $\nabla^{\#}$ is the $k^{\#}$-linear span of $\nabla$. Observe that $P S^{\#}=P k^{\#}$ and $Q S^{\#}=Q k^{\#}$ are $\nabla^{\#}$-ideals of $S^{\#}$.

By integrality, there exist prime ideals $P^{\#} \subseteq Q^{\#}$ in $S^{\#}$ with $P^{\#} \cap S=P$ and $Q^{\#} \cap S=Q$. Let $P^{\dagger}$ be the largest $\nabla^{\#}$-ideal of $S^{\#}$ contained in $P^{\#}$, and note that $P S^{\#} \subseteq P^{\dagger}$. Then $P^{\dagger} \cap S=P$ and $Q S^{\#} \cap S=Q$, whence $Q S^{\#} \nsubseteq P^{\dagger}$. By Lemma 3.1(i), there exists an element $a \in Q S^{\#}-P^{\dagger}$ such that $k^{\#} a+P^{\dagger}$ is invariant under $\Delta$ and $d_{r}(a) \in P^{\dagger}$ for all $r \in R^{\#}$. Then there are $\lambda_{1}, \ldots, \lambda_{n} \in k^{\#}$ such that $\delta_{i}(a)-\lambda_{i} a \in P^{\dagger}$ for $i=1, \ldots, n$. In particular, $\delta_{i}(a)-\lambda_{i} a \in P^{\#}$ for $i=1, \ldots, n$ and $d_{r}(a) \in P^{\#}$ for all $r \in R^{\#}$.

Choose a finite extension field $k^{*}$ of $k$ inside $k^{\#}$ such that $\lambda_{1}, \ldots, \lambda_{n} \in k^{*}$ and $a \in Q k^{*}$.

Set $R^{*}=R k^{*}$ and $S^{*}=S k^{*}$ etc., as in the first paragraph of the proof. If $P^{*}=P^{\#} \cap S^{*}$ and $Q^{*}=Q^{\#} \cap S^{*}$, then $P^{*} \subseteq Q^{*}$ are prime ideals of $S^{*}$ with $P^{*} \cap S=P$ and $Q^{*} \cap S=Q$. By integrality, $P^{*}$ is minimal over $P S^{*}=P k^{*}$ and $Q^{*}$ is minimal over $Q S^{*}=Q k^{*}$; observe also that $P S^{*}$ and $Q S^{*}$ are $\nabla^{*}$-ideals of $S^{*}$. Since $k^{*}$ is a finite field extension of $k$, the ring $R^{*}$ is 
noetherian. Hence, we may apply Corollary 2.4 and conclude that $P^{*}$ and $Q^{*}$ are $\nabla^{*}$-ideals of $S^{*}$.

If there is a prime $\nabla^{*}$-ideal $J$ of $S^{*}$ lying strictly between $P^{*}$ and $Q^{*}$, then $J \cap S$ is a prime $\nabla$-ideal of $S$ lying strictly between $P$ and $Q$, contradicting the assumption that $\nabla$-ht $(Q / P)=1$. Hence, $\nabla^{*}$-ht $\left(Q^{*} / P^{*}\right)=1$.

Now $a \in Q k^{*} \subseteq Q^{*}$. On the other hand, since $P^{*}$ is a $\nabla^{*}$-ideal of $S^{*}$ we see that $P^{*} S^{\#}$ is a $\nabla^{\#}$-ideal of $S^{\#}$ contained in $P^{\#}$, whence $P^{*} S^{\#} \subseteq P^{\dagger}$. Thus $a \notin P^{*}$. We also have

$$
\delta_{i}(a)-\lambda_{i} a \in P^{\#} \cap S^{*}=P^{*}
$$

for $i=1, \ldots, n$, and likewise $d_{r}(a) \in P^{*}$ for all $r \in R^{*}$. Thus, by Lemma 3.1(ii), the set $P^{*}+S^{*} a$ is a $\nabla^{*}$-ideal of $S^{*}$.

Let $I$ be a prime ideal of $S^{*}$ contained in $Q^{*}$ and minimal over $P^{*}+S^{*} a$. Then, by using Corollary 2.4 again, $I$ is a $\nabla^{*}$-ideal. Since $\nabla^{*}-h \mathrm{ht}\left(Q^{*} / P^{*}\right)=1$, this forces $I=Q^{*}$, so that $Q^{*}$ is a prime minimal over $P^{*}+S^{*} a$. Therefore $\operatorname{ht}\left(Q^{*} / P^{*}\right)=1$, by the Principal Ideal Theorem.

Consequently, ht $(Q / P)=1$, by integrality.

We shall say that a ring $R_{0}$ equipped with a set $\Delta_{0}$ of derivations is $\Delta_{0}$ catenary if for any two $\Delta_{0}$-prime ideals $P \subseteq Q$ in $R_{0}$, all saturated chains of $\Delta_{0}$-prime ideals between $P$ and $Q$ have the same length.

Theorem 3.3. Let $k$ be a field of characteristic zero, $R$ a commutative noetherian $k$-algebra, and $\Delta=\left\{\delta_{1}, \ldots, \delta_{n}\right\}$ a finite set of commuting $k$-linear derivations acting locally finitely on $R$. If the polynomial ring $S=R\left[x_{1}, \ldots, x_{n}\right]$ is either catenary or $\Delta$-catenary, then the differential operator ring $T=R[\Theta ; \Delta]$ is catenary.

Proof. It is enough, by Theorem 2.5 , to show that for any two prime $\nabla$-ideals $P \subseteq Q$ in $S$, all saturated chains of prime $\nabla$-ideals between $P$ and $Q$ have the same length. However, Lemma 3.2 shows that any saturated chain of prime $\nabla$-ideals between $P$ and $Q$ is in fact a saturated chain of prime ideals, and hence also a saturated chain of $\Delta$-prime ideals. (Recall from Lemma 2.2(ii) that all $\Delta$-prime ideals of $S$ are prime.) The result follows since $S$ is either catenary or $\Delta$-catenary.

Bell and Sigurdsson [2, Example 2.10] have shown that if $k$ is a field of characteristic zero and $\delta$ is the derivation $2 y z \partial / \partial x+\left(x+y^{2}\right) \partial / \partial y$ acting on the polynomial ring $R=k[x, y, z]$, then $T=R[\theta ; \delta]$ is not catenary. Hence, some restriction such as local finiteness is necessary in Theorem 3.3. The restriction that $S$ be catenary or $\Delta$-catenary is also necessary, since if the derivations in $\Delta$ are all zero then $T \cong S$. Bell and Sigurdsson have also obtained the above theorem in the case of a single locally finite derivation (see [2, Corollary 2.3, Lemma 2.7, Theorem 2.8]).

Corollary 3.4. Let $k$ be a field of characteristic zero. If $R$ is a universally catenary commutative noetherian $k$-algebra, and $\Delta$ is a finite set of commuting 
$k$-linear derivations acting locally finitely on $R$, then the differential operator ring $T=R[\Theta ; \Delta]$ is universally catenary.

Proof. Observe that any polynomial ring $T\left[x_{n+1}, \ldots, x_{q}\right]$ is isomorphic to $R\left[\theta_{1}, \ldots, \theta_{q} ; \delta_{1}, \ldots, \delta_{q}\right]$ where $\delta_{n+1}, \ldots, \delta_{q}$ all equal the zero derivation on $R$. Since $R$ is assumed to be universally catenary, the polynomial ring $R\left[x_{1}, \ldots, x_{q}\right]$ is catenary, and so Theorem 3.3 shows that $T\left[x_{n+1}, \ldots, x_{q}\right]$ is catenary.

In the setting of Corollary 3.4, suppose that $R$ is affine over $k$, and let $d(A)$ denote the Gelfand-Kirillov dimension of any $k$-algebra $A$ [11]. It can be deduced from Theorem 2.5 and Lemma 3.2 that the generalization of Tauvel's height formula discussed in [12] is valid in $T$. Namely, if $P, Q$ are prime ideals of $T$ with $P \subseteq Q$ then

$$
\operatorname{ht}(Q / P)=d(T / P)-d(T / Q) .
$$

Of course, this also follows from Gabber's work [6], because when $R$ is affine and $\Delta$ acts locally finitely, $T$ is a homomorphic image of the enveloping algebra of a finite-dimensional solvable Lie algebra.

A similar result is valid in the positive characteristic context of Theorem 4.1, inasmuch as $T$ is in this case an affine PI-algebra, and so Schelter's formula [14, Theorem 4.4.27] applies.

\section{Catenarity IN CHARACTERISTIC $p$}

We continue to study catenarity in a differential operator ring $T=R[\Theta ; \Delta]$ over a commutative noetherian ring $R$. Notation N1 will be assumed throughout the section. A number of problems arise in the case of positive characteristic that are not present in the characteristic zero case, but we can still proceed along a route roughly parallel to that taken in the previous section. The case in which $R$ is affine over a field of characteristic $p$ is quite easy, since then $T$ is an affine PI-algebra and catenarity follows immediately. Hence, we begin with this case since it does not require the more general manipulations used later.

Theorem 4.1. Let $k$ be a field of characteristic $p>0$ and suppose that $R$ is an affine commutative algebra over $k$. Let $\Delta=\left\{\delta_{1}, \ldots, \delta_{n}\right\}$ be a finite set of commuting $k$-linear derivations on $R$ and set $T=R[\Theta ; \Delta]$. Then $T$ is an affine PI-algebra and hence is universally catenary.

Proof. Suppose that $R=k\left[a_{1}, \ldots, a_{m}\right]$. Set

$$
C=\left\{r \in R \mid \delta_{i}(r)=0 \text { for } i=1, \ldots, n\right\} ;
$$

then $C[\theta]$ is a commutative subalgebra of $T$. Now $\delta_{i}\left(a_{j}^{p}\right)=0$ for all $i, j$, and hence $k\left[a_{1}^{p}, \ldots, a_{n}^{p}\right] \subseteq C$. Consequently, $T$ is generated as a right $C[\Theta]$ module by the finite set

$$
\left\{a_{1}^{s(1)} a_{2}^{s(2)} \ldots a_{m}^{s(m)} \mid s(1), \ldots, s(m)=0, \ldots, p-1\right\} .
$$


Hence, since $T$ embeds in $\operatorname{End}\left(T_{C[\Theta]}\right)$ via left multiplication, $T$ is a homomorphic image of a $C[\Theta]$-subalgebra of a matrix algebra $M_{q}(C[\Theta])$. Therefore $T$ is an affine PI-algebra over $k$, as are all finite polynomial extensions of $T$, and the results follows [15, Theorem 4; 14, Theorem 4.4.27].

We now present two examples illustrating the misbehavior of the $\Phi$-correspondence in characteristic $p$.

Example 4.2. Let $k$ be a field of characteristic 2 and $t$ an indeterminate, set $R=k[t] /\left(t^{2}\right)$, and let $z$ denote the image of $t$ in $R$. Since $d\left(t^{2}\right) / d t=2 t=0$, the derivation $d / d t$ on $k[t]$ induces a $k$-linear derivation $\delta$ on $R$ such that $\delta(z)=1$. Set $T=R[\theta ; \delta]$. We show that $P=T \theta^{2}$ is a maximal ideal of $T$ and that $\Phi(P)$ is not a $\delta$-prime ideal in $S=R[x]$. Thus, even when $R$ is an affine algebra, $\Phi(P)$ need not be a prime ideal.

The element $\theta^{2}$ is central in $T$ because $\delta^{2}=0$; hence, $P$ is an ideal of $T$. If $I$ is an ideal of $T$ properly containing $P$, choose an element $a+b \theta \in I$ where $a, b \in R$, not both zero. In case $b \neq 0$, observe that $I$ contains the element $[a+b \theta, z]=b$. Now $I$ contains a nonzero element of $R$, say $\alpha+\beta z$ with $\alpha, \beta \in k$, not both zero. If $\beta \neq 0$, observe that $I$ contains the element $[\theta, \alpha+\beta z]=\beta$. Thus $I$ contains a nonzero element of $k$, and so $I=T$. Hence, $P$ is a maximal ideal of $T$.

Now $\Phi(P)=S x^{2}$. Since $\delta(x)=0$ the ideal $S x$ is a $\delta$-ideal of $S$ such that $(S x)^{2} \subseteq \Phi(P)$, while $S x \nsubseteq \Phi(P)$. Therefore $\Phi(P)$ is not a $\delta$-prime ideal of $S$ (nor even a $\delta$-semiprime ideal).

In this example, $d_{z}=-d / d x$, which makes it clear why $S x$ is not a $\nabla$-ideal of $S$ (that of course being necessary since $\Phi(P)$ must be a $\nabla$-prime ideal).

In general, if a set $\Delta_{0}$ of derivations acts on a noetherian ring $R_{0}$, then the (prime) radical of any $\Delta_{0}$-prime ideal of $R_{0}$ is a prime ideal (Lemma 2.2(i)). One might hope that the same would be true of $\nabla$-prime ideals in $S$ (and indeed this holds in the previous example). The next example shows that this is not true in general.

Example 4.3. Let $l$ be a field of characteristic 2 and $t, u$ independent indeterminates. Set $k=l\left(t^{2}\right)$ and $R=l(t)[u] /\left(u^{2}\right)$, and let $z$ denote the image of $u$ in $R$. Then $R$ is a four-dimensional $k$-algebra, and the derivation $t \partial / \partial t+t \partial / \partial u$ on $l(t)[u]$ induces a $k$-linear derivation $\delta$ on $R$ such that $\delta(t)=\delta(z)=t$. Set $T=R[\theta ; \delta]$. We show that $P=T\left(\theta^{2}+\theta\right)$ is a maximal ideal of $T$ and that the radical of $\Phi(P)$ is not a prime ideal in $S=R[x]$.

Since $\delta^{2}+\delta=0$, the element $\theta^{2}+\theta$ is central in $T$, and so $P$ is an ideal of $T$. If $I$ is an ideal of $T$ properly containing $P$, choose an element $a+b \theta \in I$ where $a, b \in R$, not both zero. In case $b \neq 0$, observe that $I$ contains the element $[a+b \theta, z]=b t$. Now $I$ contains a nonzero element of $R$, say $\alpha+\beta z$ with $\alpha, \beta \in l(t)$, not both zero. If $\alpha \neq 0$ then $\alpha+\beta z$ is invertible in $R$ and 
$I=T$. If $\alpha=0$ then $\beta \neq 0$ and $z \in I$. In this case, $t=[\theta, z] \in I$ and again $I=T$. Thus $P$ is a maximal ideal of $T$.

Now $\Phi(P)=S\left(x^{2}+x\right)$. If $J=S\left(x^{2}+x\right)+S z$, then $J / \Phi(P)$ is nilpotent and $S / J$ is isomorphic to $l(t)[x] /\left(x^{2}+x\right)$. Thus $J$ is the radical of $\Phi(P)$, and $J$ is not a prime ideal of $S$.

Theorem 4.4. Let $R$ be a commutative noetherian ring, let $\Delta$ be a finite set of commuting derivations on $R$, and set $T=R[\Theta ; \Delta]$. Let $P$ be a prime ideal of $T$ such that $\operatorname{char}(T / P)=p>0$. Then any nonzero ideal of $T / P$ contains $a$ central non-zero-divisor.

Proof. After replacing $T$ by $T /(P \cap R) T$, we may assume that $P \cap R=0$. Then $R$ is a $\Delta$-prime ring of characteristic $p$. By Lemma $2.2(\mathrm{i})$, there is a unique minimal prime ideal in $R$, say $N$; then $N$ is the prime radical of $R$ and so is nilpotent. Now $R-N$ is a right and left Ore set in $T$ disjoint from $P$. As $T$ is noetherian it follows that $R-N \subseteq \mathscr{C}(P)$ [3, Proof of Satz 2.10].

Let $A$ be an ideal of $T$ that properly contains $P$. Let $I$ be the minimal degree (with respect to the Dixmier ordering) for elements of $A-P$, and choose $a \in A-P$ with degree $I$ and leading coefficient $\alpha$. Define

$$
\begin{aligned}
& A^{\circ}=\{0\} \cup\{\text { leading coefficients of elements of } A \text { of degree } I\}, \\
& P^{\circ}=\{0\} \cup\{\text { leading coefficients of elements of } P \text { of degree } I\} .
\end{aligned}
$$

It is clear that $P^{\circ} \subseteq A^{\circ}$ and that these are $\Delta$-ideals of $R$. Then $\alpha \in A^{\circ}$ by definition of $A^{\circ}$, and $\alpha \notin P^{\circ}$ since if there existed $b \in P$ with degree $I$ and leading coefficient $\alpha$, then $a-b$ would be an element of $A-P$ with degree less than $I$.

Suppose that $P^{\circ} \nsubseteq N$, say there exists $\beta \in P^{\circ}-N$. Then there exists $b \in P$ with degree $I$ and leading coefficient $\beta$. Now $\beta a-\alpha b$ is an element of $A$ with degree less than $I$, whence $\beta a-\alpha b \in P$ and so $\beta a \in P$. However, as $\beta \in R-N$ and $a \in T-P$, this contradicts the fact that $R-N \subseteq \mathscr{C}(P)$. Thus $P^{\circ} \subseteq N$, and hence $P^{0}$ is nilpotent. Since $R$ is $\Delta$-prime, it follows that $P^{\circ}=0$.

On the other hand, $A^{\circ}$ is a nonzero $\Delta$-ideal of $R$, whence $A^{\circ}$ is not nilpotent, and as $R$ is noetherian, $A^{\circ}$ cannot even be nil. Choose a nonnilpotent element $\gamma \in A^{\circ}$. Then $\gamma^{p} \neq 0$, and there exists $u \in A$ with degree $I$ and leading coefficient $\gamma^{p}$; moreover, $u \notin P$ because $P^{\circ}=0$.

For $i=1, \ldots, n$, observe that $\delta_{i}\left(\gamma^{p}\right)=0$ and so $\left[\theta_{i}, u\right]$ is an element of $A$ with degree less than $I$, whence $\left[\theta_{i}, u\right] \in P$. For $r \in R$, Lemma 1.5(i) shows that $[r, u]$ has lower total degree than $u$, whence $[r, u]$ has Dixmier degree less than $I$, and so $[r, u] \in P$. Therefore $u+P$ is a nonzero central element of $T / P$. Finally, since $T / P$ is a prime ring, $u+P$ must be a non-zero-divisor $T / P$.

Theorem 4.4 is parallel to [1, Proposition 6.2] but does not follow from it, since our coefficient ring $R$ need not be $\Delta$-hypercentral. However, with 
an appropriately weaker version of $\Delta$-hypercentrality, Theorem 4.4 could be proved using an induction argument similar to the proof of [1, Proposition 6.2].

We shall use Theorem 4.4 as an analog of Lemma 3.1 in proving catenarity in characteristic $p$. For the remainder of the section, we assume Notation N1. The next step is an analog of Lemma 3.2, but since in characteristic $p$ the $\nabla$-prime ideals of $S$ are not always prime, we must work with prime ideals minimal over $\nabla$-prime ideals.

Proposition 4.5. Assume that $k$ is a field of characteristic $p>0$ and that $R$ is a commutative noetherian $k$-algebra. Let $P \subseteq Q$ be prime ideals of $T$ such that $\mathrm{ht}(Q / P)=1$, and let $A \subseteq B$ be prime ideals of $S$ such that $A$ is minimal over $\Phi(P)$ and $B$ is minimal over $\Phi(Q)$. Then $\operatorname{ht}(B / A)=1$.

Proof. By Theorem 4.4 there exists an element $c \in Q$ which is a central nonzero-divisor modulo $P$. Set $u=\Phi(c)$ and note that $u \in \Phi(Q)-\Phi(P)$. Now $[c, R] \subseteq P$ and hence it follows from Proposition 1.6(iii) that $u$ is a nonzero-divisor modulo $\Phi(P)$, and that $\Phi(P+c T)=\Phi(P)+u S$. Since $u$ is a non-zero-divisor modulo $\Phi(P)$, we see that $u \notin A$, and so $B \neq A$. Since $P+c T$ is an ideal of $T$ and $\Phi(P+c T)=\Phi(P)+u S$, it follows that $\Phi(P)+u S$ is a $\nabla$-ideal of $S$, contained in $\Phi(Q)$ and properly containing $\Phi(P)$.

We claim that $B$ is minimal over $A+u S$. If not, there is a prime ideal $I$ containing $A+u S$ and strictly contained in $B$. Let $E$ be the largest $\nabla$-ideal contained in $I$; then $E$ is a $\nabla$-prime ideal. Since $\Phi(P)+u S \subseteq A+u S \subseteq I$, we have $\Phi(P)+u S \subseteq E$, and so $E$ properly contains $\Phi(P)$. By Corollary 1.10, $\Phi(Q)$ is the largest $\nabla$-ideal contained in $B$, and so from $E \subseteq I \subseteq B$ we obtain $E \subseteq \Phi(Q)$. If $E=\Phi(Q)$ then $\Phi(Q) \subseteq I$, contradicting the minimality of $B$. Thus $\Phi(Q)$ properly contains $E$.

Now by Corollary $1.8, \Phi^{-1}(E)$ is a prime ideal of $T$ lying strictly between $P$ and $Q$, but that contradicts the assumption that $\mathrm{ht}(Q / P)=1$. Hence, $B$ is minimal over $A+u S$, as claimed. Therefore, by the Principal Ideal Theorem, $\operatorname{ht}(B / A)=1$.

Theorem 4.6. Let $k$ be a field of characteristic $p>0$, let $R$ be a commutative noetherian $k$-algebra, and let $\Delta=\left\{\delta_{1}, \ldots, \delta_{n}\right\}$ be a finite set of commuting $k$ linear derivations on $R$. If the polynomial ring $S=R\left[x_{1}, \ldots, x_{n}\right]$ is catenary, then the differential operator ring $T=R[\Theta ; \Delta]$ is catenary.

Proof. Given prime ideals $P \subseteq Q$ in $T$, we need to show that all saturated chains of prime ideals between $P$ and $Q$ have the same length. To prove this, we may replace $P$ by any minimal prime ideal contained in $P$, and so, without loss of generality, we may assume that $P$ is minimal. After factoring out $P \cap R$, we may also assume that $P \cap R=0$. Now $R$ is a $\Delta$-prime ring, and so $T$ is a prime ring. Hence, as $P$ is a minimal prime, $P=0$.

Since $R$ is a noetherian $\Delta$-prime ring, $R$ has a unique minimal prime ideal, say $N$, by Lemma 2.2(i). Then $N S$ is the unique minimal prime ideal of $S$. 
Choose a prime ideal $Q_{0}$ in $S$ minimal over $\Phi(Q)$. Since $S$ is assumed to be catenary, all saturated chains of prime ideals between $Q_{0}$ and $N S$ have the same length, namely $\mathrm{ht}\left(Q_{0}\right)$. We shall prove that if

$$
Q=P_{0}>P_{1}>\cdots>P_{d}=P=0
$$

is any saturated chain of prime ideals between $Q$ and $P$, then $d=\operatorname{ht}\left(Q_{0}\right)$.

Construct a chain of prime ideals in $S$ as follows: Start with $Q_{0}$, and, once $Q_{i}$ has been chosen, let $Q_{i+1}$ be a prime ideal contained in $Q_{i}$ and minimal over $\Phi\left(P_{i+1}\right)$. By Proposition 4.5,

$$
Q_{0}>Q_{1}>\cdots>Q_{d}=N S
$$

is a saturated chain of prime ideals in $S$, and therefore $d=\operatorname{ht}\left(Q_{0}\right)$, as desired.

Corollary 4.7. Let $k$ be a field of characteristic $p>0$. If $R$ is a universally catenary commutative noetherian $k$-algebra, and $\Delta$ is a finite set of commuting $k$-linear derivations on $R$, then the differential operator ring $T=R[\Theta ; \Delta]$ is universally catenary.

In many cases where catenarity can be proved, such as for commutative affine domains, one can also prove that all maximal ideals have the same height. We shall prove this for prime factor rings of the differential operator ring $T$ in the affine case of our current hypotheses.

Theorem 4.8. Let $k$ be a field of characteristic $p>0$ and suppose that $R$ is an affine commutative algebra over $k$. Let $\Delta=\left\{\delta_{1}, \ldots, \delta_{n}\right\}$ be a finite set of commuting $k$-linear derivations on $R$ and set $T=R[\Theta ; \Delta]$. If $P$ is any prime ideal of $T$, then all maximal ideals of $T / P$ have the same height. In particular, if $R$ is a $\Delta$-prime ring, then all maximal ideals of $T$ have height $\mathrm{K} \cdot \operatorname{dim}(R)+n$. Proof. We show that all saturated chains of prime ideals from $P$ to any maximal ideal have the same length. As in the proof of Theorem 4.6, we may assume that $R$ is a $\Delta$-prime ring and $P=0$. It remains to show that if $M$ is any maximal ideal of $T$ and

$$
M=P_{0}>P_{1}>\cdots>P_{d}=0
$$

is any saturated chain of prime ideals between $M$ and 0 , then $d=\mathrm{K} \cdot \operatorname{dim}(R)+$ $n$.

Since $R$ is a noetherian $\Delta$-prime ring, $R$ has a unique minimal prime ideal, say $N$, by Lemma 2.2(i). Then $N S$ is the unique minimal prime ideal of $S$. Construct a chain of prime ideals in $S$ as follows: Let $Q_{0}$ be a prime ideal minimal over $\Phi\left(P_{0}\right)$, and, once $Q_{i}$ has been chosen, let $Q_{i+1}$ be a prime ideal contained in $Q_{i}$ and minimal over $\Phi\left(P_{i+1}\right)$. By Proposition 4.5,

$$
Q_{0}>Q_{1}>\cdots>Q_{d}=N S
$$

is a saturated chain of prime ideals in $S$. Since $S$ is catenary [13, Corollary 3 , $(14 . \mathrm{H})], d=\mathrm{ht}\left(Q_{0} / N S\right)$. 
By Theorem 4.1, $T / M$ is an affine PI-algebra. As $T / M$ is also simple, it must be finite-dimensional over $k$, and since $\Phi$ is a $k$-linear isomorphism the same is true for $S / \Phi(M)$. Thus $S / Q_{0}$ is finite-dimensional, whence $Q_{0}$ is a maximal ideal of $S$. Now all maximal ideals of $S / N S$ have the same height, namely $\mathrm{K} \cdot \operatorname{dim}(R)+n[13$, Corollary $3,(14 . \mathrm{H})]$. Therefore

$$
d=\operatorname{ht}\left(Q_{0} / N S\right)=\mathrm{K} \cdot \operatorname{dim}(R)+n .
$$

Theorem 4.8 does not hold in characteristic zero, as the following example (the enveloping algebra of the 3-dimensional Heisenberg algebra) shows. Note that the derivation $\delta$ in this example is locally finite.

Example 4.9. Let $k$ be a field of characteristic zero and $u, v$ independent indeterminates, let $R=k[u, v]$ and $\delta=v \partial / \partial u$, and set $T=R[\theta ; \delta]$. We show that $M_{1}=(v-1) T$ and $M_{2}=u T+v T+\theta T$ are maximal ideals of $T$ such that $\operatorname{ht}\left(M_{1}\right)=1$ while $\operatorname{ht}\left(M_{2}\right)=3$.

Since $\delta(v-1)=0$, we see that $M_{1}$ is an ideal of $T$ and that

$$
T / M_{1} \cong k[u][\theta ; d / d u] \cong A_{1}(k) .
$$

Hence, $T / M_{1}$ is a simple ring and $M_{1}$ is a maximal ideal of $T$. On the other hand, $u T+v T$ is an ideal of $T$ and $T /(u T+v T) \cong k[\theta]$, whence $M_{2}$ is a maximal ideal of $T$.

If $P_{1}$ is a nonzero prime ideal contained in $M_{1}$, then $P_{1} \cap R$ is a nonzero prime ideal of $R$ (because $\delta \neq 0$ and $\operatorname{char}(k)=0)$. Since $P_{1} \cap R \subseteq M_{1} \cap R=$ $(v-1) R$ and $(v-1) R$ has height 1 , it follows that $P_{1} \cap R=(v-1) R$ and $P_{1}=M_{1}$. Thus $\operatorname{ht}\left(M_{1}\right)=1$.

Since $M_{2}>u T+v T>v T>0$ is a chain of prime ideals of $T$, we have $\mathrm{ht}\left(M_{2}\right) \geq 3$. On the other hand, $\mathrm{K} \cdot \operatorname{dim}(R)=2$ and so $\mathrm{K} \cdot \operatorname{dim}(T) \leq 3$. Therefore $\operatorname{ht}\left(M_{2}\right)=3$.

Alternatively, since $\delta$ acts locally finitely on $R$, the heights of $M_{1}$ and $M_{2}$ can be obtained from Lemma 3.2 and the observation that $\Phi\left(M_{1}\right)=(v-1) S$ while $\Phi\left(M_{2}\right)=u S+v S+S x$.

\section{AfFine $\mathbb{Z}$-ALGEBras}

In this section we combine the results of $\S \S 3$ and 4 to obtain a catenarity result for differential operator rings over affine $\mathbb{Z}$-algebras. Throughout the section, we assume that $R$ is a commutative ring finitely generated as an algebra over $\mathbb{Z}$, and we assume Notation $\mathrm{N} 1$.

In the present context, we say that $\Delta$ acts locally finitely provided each element of $R$ is contained in a $\Delta$-invariant additive subgroup of $R$ of finite torsionfree rank. (Recall that the torsionfree rank of an abelian group $G$ is the dimension of the $\mathbb{Q}$-vector space $\mathbb{Q} \otimes_{\mathbf{Z}} G$.)

Proposition 5.1. Assume that $\Delta$ acts locally finitely on $R$. Let $P \subseteq Q$ be prime ideals of $T$ such that $\mathrm{ht}(Q / P)=1$, and let $A \subseteq B$ be prime ideals of $S$ such that $A$ is minimal over $\Phi(P)$ and $B$ is minimal over $\Phi(Q)$. Then $\operatorname{ht}(B / A)=1$. 
Proof. We consider three possible cases.

Case (i). Suppose that $\operatorname{char}(T / Q)=0$. Then $Q \cap \mathbb{Z} \cdot 1=0$ and $B \cap \mathbb{Z} \cdot 1=0$. By localizing with respect to $\mathbb{Z} \cdot 1-\{0\}$ in $R, S, T$, we may replace these rings by $\mathbb{Q} \otimes_{\mathbf{Z}} R, \mathbb{Q} \otimes_{\mathbf{Z}} S, \mathbb{Q} \otimes_{\mathbf{Z}} T$ respectively. Now $R$ is a commutative noetherian algebra over $Q$ and $\Delta$ acts locally finitely in the sense of $\S 3$. The result now follows from Corollary 2.4 and Lemma 3.2.

Case (ii). Suppose that $\operatorname{char}(T / P)=p>0$. Then $p T \subseteq P$ and $p S \subseteq A$. Replace $R, S, T$ by $R / p R, S / p S, T / p T$ respectively. Now $R$ is a commutative noetherian algebra over $\mathbb{Z} / p \mathbb{Z}$, and the result follows from Proposition 4.5.

Case (iii). Suppose that $\operatorname{char}(T / P)=0$ while $\operatorname{char}(T / Q)=p>0$. Now the element $p \cdot 1$ in $T$ is an element of $Q$ which is a central non-zero-divisor modulo $P$, and we may use the proof of Proposition 4.5, with the element $c$ replaced by $p \cdot 1$.

Theorem 5.2. Let $R$ be a commutative affine $\mathbb{Z}$-algebra, let $\Delta=\left\{\delta_{1}, \ldots, \delta_{n}\right\}$ be a finite set of commuting derivations acting locally finitely on $R$, and set $T=R[\Theta ; \Delta]$. Then $T$ is universally catenary, and if $P$ is any prime ideal of $T$, all maximal ideals of $T / P$ have the same height. In particular, if $R$ is a $\Delta$-prime ring then all maximal ideals of $T$ have height $\mathrm{K} . \operatorname{dim}(R)+n$.

Proof. As in the proof of Corollary 3.4, a polynomial extension of $T$ is isomorphic to a differential operator ring over a polynomial extension of $R$, and so it is enough to prove catenarity in place of universal catenarity.

For the first two conclusions, it suffices to show that given a prime ideal $P$ in $T$, all saturated chains of prime ideals from $P$ to any maximal ideal have the same length. As in the proof of Theorem 4.6, it is enough to consider the case where $R$ is $\Delta$-prime and $P=0$. Thus the entire theorem will be proved if we assume that $R$ is $\Delta$-prime and show that for any maximal ideal $M$ of $T$, all saturated chains of prime ideals between 0 and $M$ have length $\mathrm{K} \cdot \operatorname{dim}(R)+n$.

We proceed as in the proof of Theroem 4.8, using Proposition 5.1 in place of Proposition 4.5. We need to know that $S$ is catenary, that $Q_{0}$ is a maximal ideal of $S$, and that all maximal ideals in $S / N S$ have height $\mathrm{K} \cdot \operatorname{dim}(R)+n$. The first and third statements follow from [13, Theorem 33, (16.D)] and [10, Exercise 3, p. 114]. For the second, $M \cap \mathbb{Z} \cdot 1 \neq 0$, since $M$ is a primitive ideal [8, Theorem 2, p. 271]. Thus $T / M$ is a simple PI-algebra, affine over $\mathbb{Z} / p \mathbb{Z}$ for some $p>0$, and so $T / M$ is finite. Hence, $S / \Phi(M)$ and $S / Q_{0}$ are finite, and therefore $Q_{0}$ is a maximal ideal of $S$.

\section{Partial CATENARity in ChaRACTERistic Zero}

In this final section we return to a differential operator ring $T$ over a commutative noetherian $\mathbb{Q}$-algebra $R$ and study catenarity for pairs of prime ideals in $T$ which have the same contraction in $R$. 
Theorem 6.1. Let $K$ be a division ring of characteristic zero, let $\Delta=$ $\left\{\delta_{1}, \ldots, \delta_{n}\right\}$ be a finite set of commuting derivations on $K$, and set $T=$ $R[\Theta ; \Delta]$.

(i) If $P$ is a prime ideal of $T$, there is a right and left Ore set $\mathscr{C} \subseteq \mathscr{C}(P)$ such that $T \mathscr{C}^{-1}$ is isomorphic to a polynomial ring over a division ring.

(ii) $T$ is universally catenary.

Proof. (i) Certainly $P \cap K=0$. Choose a subset $\Theta^{\prime} \subseteq\left\{\theta_{1}, \ldots, \theta_{n}\right\}$ maximal with respect to the property that $P \cap K\left[\Theta^{\prime}\right]=0$. After renumbering, we may assume that $\Theta^{\prime}=\left\{\theta_{1}, \ldots, \theta_{t}\right\}$ for some $t$. Then $P \cap K\left[\theta_{1}, \ldots, \theta_{t}\right]=0$ and $P \cap K\left[\theta_{1}, \ldots, \theta_{t}, \theta_{j}\right] \neq 0$ for $j=t+1, \ldots, n$. Observe that since

$$
T=K\left[\theta_{1}, \ldots, \theta_{t} ; \delta_{1}, \ldots, \delta_{t}\right]\left[\theta_{t+1}, \ldots, \theta_{n} ; \delta_{t+1}, \ldots, \delta_{n}\right],
$$

the set $\mathscr{C}=K\left[\theta_{1}, \ldots, \theta_{t}\right]-\{0\}$ is a right and left Ore set in $T$. Moreover, $\mathscr{C}$ is disjoint from $P$, and as $T$ is noetherian it follows that $\mathscr{C} \subseteq \mathscr{C}(P)$ [3, Proof of Satz 2.10]. We identify $T \mathscr{C}^{-1}$ with the differential operator ring

$$
D\left[\theta_{t+1}, \ldots, \theta_{n} ; \delta_{t+1}, \ldots, \delta_{n}\right],
$$

where $D$ is the quotient division ring of $K\left[\theta_{1}, \ldots, \theta_{t}\right]$ and $\delta_{t+1}, \ldots, \delta_{n}$ have been extended to $D$ via [3, Lemma 4.1].

For $j \in\{t+1, \ldots, n\}$ we have $P \cap D\left[\theta_{j}\right] \neq 0$ and so $P \mathscr{C}^{-1} \cap D\left[\theta_{j}\right] \neq 0$. Hence, $D\left[\theta_{j} ; \delta_{j}\right]$ is not a simple ring. Therefore $\delta_{j}$ must be an inner derivation on $D$ [3, Satz 4.7], and so there exists $d_{j} \in D$ such that the element $z_{j}=\theta_{j}-d_{j}$ commutes with $D$. Note that $D\left[\theta_{j}\right]=D\left[z_{j}\right]$.

For $i, j \in\{t+1, \ldots, n\}$ we have

$$
\left[z_{i}, z_{j}\right]=\left[\theta_{i}-d_{i}, \theta_{j}-d_{j}\right]=-\delta_{i}\left(d_{j}\right)+\delta_{j}\left(d_{i}\right)+\left[d_{i}, d_{j}\right] \in D,
$$

and so either $\left[z_{i}, z_{j}\right]=0$ or $D\left[\theta_{i}, \theta_{j}\right]=D\left[z_{i}, z_{j}\right] \cong A_{1}(D)$. However, $A_{1}(D)$ is a simple ring, whereas $D\left[\theta_{i}, \theta_{j}\right]$ is not simple since $P \mathscr{C}^{-1} \cap D\left[\theta_{i}, \theta_{j}\right] \neq 0$. Hence, $\left[z_{i}, z_{j}\right]=0$. Therefore $z_{t+1}, \ldots, z_{n}$ are commuting indeterminates over $D$, and

$$
T \mathscr{C}^{-1}=D\left[\theta_{t+1}, \ldots, \theta_{n}\right]=D\left[z_{t+1}, \ldots, z_{n}\right]
$$

is a polynomial ring over $D$.

[On the side, we note that it follows that $P \mathscr{C}^{-1}$ has a central set of generators in $T \mathscr{C}^{-1}$, whence $P \mathscr{C}^{-1}$ is localizable [17, Corollary 1, p. 45], and consequently $P$ is localizable. Alternatively, [1, Theorem 7.7$]$ implies that $P$ is localizable.]

(ii) As in the proof of Corollary 3.4, it is enough to prove that $T$ is catenary.

Let $Q \subseteq P$ be prime ideals of $T$. By (i), there is an Ore set $\mathscr{C} \subseteq \mathscr{C}(P)$ such that $T \mathscr{C}^{-1}$ is isomorphic to a polynomial ring $D\left[x_{t+1}, \ldots, x_{n}\right]$ over a division ring $D$. Now

$$
T \mathscr{C}^{-1} \cong D \otimes_{C} C\left[x_{t+1}, \ldots, x_{n}\right],
$$

where $C$ is the center of $D$. Since $D$ is a central simple $C$-algebra, extension and contraction provide inverse bijections between the ideals of $C\left[x_{t+1}, \ldots, x_{n}\right]$ 
and the ideals of $T \mathscr{C}^{-1}$ [4, Theorem 2, p. 363], and hence extension and contraction provide inverse bijections between the prime ideals of these two rings. Since $C\left[x_{t+1}, \ldots, x_{n}\right]$ is catenary, it follows that $T \mathscr{C}^{-1}$ is catenary. By [3, Satz 2.10], contraction and extension provide inverse bijections between the prime ideals of $T \mathscr{C}^{-1}$ and those prime ideals of $T$ disjoint from $\mathscr{C}$. Therefore all saturated chains of prime ideals between $Q$ and $P$ have the same length.

Corollary 6.2. Let $R$ be a commutative noetherian $\mathbb{Q}$-algebra, let $\Delta$ be a finite set of commuting derivations on $R$, and set $T=R[\Theta ; \Delta]$. If $P \supseteq Q$ are prime ideals of $T$ such that $P \cap R=Q \cap R$, then all saturated chains of prime ideals between $P$ and $Q$ have the same length.

Proof. The ideal $Q \cap R$ is a $\Delta$-prime ideal of $R$, and so by Lemma 2.2(ii) it is a prime ideal. Thus, by factoring out $Q \cap R$, we may assume that $R$ is a domain and that $P \cap R=Q \cap R=0$. Now the set $\mathscr{C}=R-\{0\}$ is a right and left Ore set in $T$, and we may identify $T \mathscr{C}^{-1}$ with $K[\Theta ; \Delta]$, where $K$ is the quotient field of $R$ and the derivations in $\Delta$ have been extended to $K$ via the quotient rule. Since $T \mathscr{C}^{-1}$ is catenary by Theorem 6.1 , the result follows on applying [3, Satz 2.10].

Corollary 6.2 shows that catenarity in $T$ can only fail between pairs of prime ideals with distinct contractions in $R$, and so it is bound up with properties of $\Delta$-prime ideals of $R$. This is easily seen in the Bell-Sigurdsson example, where the prime ideals in the two saturated chains of differing lengths are all induced primes. In light of this, we conclude with the following question. Suppose that $R$ is a commutative noetherian $\mathbb{Q}$-algebra equipped with a finite set $\Delta$ of commuting derivations, and $T=R[\Theta ; \Delta]$. If the corresponding polynomial ring $S=R[X]$ is both catenary and $\Delta$-catenary, must $T$ be catenary? (The answer is positive in the case of a single derivation, by [2, Corollary 2.3].)

\section{REFERENCES}

1. A. D. Bell, Localization and ideal theory in iterated differential operator rings, J. Algebra 106 (1987), 376-402.

2. A. D. Bell and G. Sigurdsson, Catenarity and Gelfand-Kirillov dimension in Ore extensions, J. Algebra (to appear).

3. W. Borho, P. Gabriel, and R. Rentschler, Primideale in Einhüllenden auflösbarer Lie-Algebren, Lecture Notes in Math., vol. 357, Springer-Verlag, Berlin, 1973.

4. P. M. Cohn, Algebra, Vol. II, Wiley, London, 1977.

5. J. R. Fisher, A Goldie theorem for differentiably prime rings, Pacific J. Math. 58 (1975) 71-77.

6. O. Gabber, Equidimensionalité de la variété charactéristique, Exposé de O. Gabber, rédigé par T. Levasseur, Université de Paris VI, 1982.

7. K. R. Goodearl and R. B. Warfield, Jr., Primitivity in differential operator rings, Math. Z. 180 (1982), 503-523.

8. R. Irving, Generic flatness and the Nullstellensatz for Ore extensions, Comm. Algebra 7 (1979), 259-277.

9. D. A. Jordan, Noetherian Ore extensions and Jacobson rings, J. London Math. Soc. 10 (1975), 281-291. 
10. I. Kaplansky, Commutative rings, Allyn and Bacon, Boston, Mass., 1970.

11. G. Krause and T. H. Lenagan, Growth of algebras and Gelfand-Kirillov dimension, Research Notes in Math., No. 116, Pitman, Boston, Mass., 1985.

12. M. Lorenz, Chains of prime ideals in enveloping algebras of solvable Lie algebras, J. London Math. Soc. (2) 24 (1981) 205-210.

13. H. Matsumura, Commutative algebra, 2nd ed., Benjamin/Cummings, Reading, Mass., 1980.

14. L. H. Rowen, Polynomial identities in ring theory, Academic Press, New York, 1980.

15. W. Schelter, Non-commutative affine P.I. rings are catenary, J. Algebra 51 (1978), 12-18.

16. G. Sigurdsson, Differential operator rings whose prime factors have bounded Goldie dimension, Arch. Math. 42 (1984), 348-353.

17. P. F. Smith, Localization and the AR property, Proc. London Math. Soc. (3) 22 (1971), 39-68.

18. S. Yammine, Les théorèmes de Cohen-Seidenberg en algèbre non commutative, Séminaire d'Algèbre P. Dubreil 1977-78, Lecture Notes in Math., vol. 740, Springer-Verlag, Berlin, 1979, pp. 120-169.

Department of Mathematics, University of Glasgow, Glasgow G12 8QW, Scotland

Department of Mathematics, University of Utah, Salt lake City, Utah 84112

Department of Mathematics, University of Edinburgh, J.C.M.B., Kings Buildings, Mayfield RoAd, Edinburgh EH9 3JZ, Scotland 